\title{
Distinct GABAergic Targets of Feedforward and Feedback Connections Between Lower and Higher Areas of Rat Visual Cortex
}

\author{
Yuri Gonchar and Andreas Burkhalter \\ Department of Anatomy and Neurobiology, Washington University School of Medicine, St. Louis, Missouri 63110
}

\begin{abstract}
Processing of visual information is performed in different cortical areas that are interconnected by feedforward (FF) and feedback (FB) pathways. Although FF and FB inputs are excitatory, their influences on pyramidal neurons also depend on the outputs of GABAergic neurons, which receive FF and FB inputs. Rat visual cortex contains at least three different families of GABAergic neurons that express parvalbumin (PV), calretinin (CR), and somatostatin (SOM) (Gonchar and Burkhalter, 1997). To examine whether pathway-specific inhibition (Shao and Burkhalter, 1996) is attributable to distinct connections with GABAergic neurons, we traced FF and FB inputs to PV, CR, and SOM neurons in layers 1-2/3 of area 17 and the secondary lateromedial area in rat visual cortex. We found that in layer 2/3 maximally $2 \%$ of FF and FB inputs go to CR and SOM neurons. This contrasts with $12-13 \%$ of FF and FB inputs onto layer $2 / 3 \mathrm{PV}$ neurons. Unlike inputs to layer 2/3, connections to layer 1, which contains CR but lacks SOM and PV somata, are pathway-specific: $21 \%$ of FB inputs go to CR neurons, whereas FF inputs to layer 1 and its CR neurons are absent. These findings suggest that FF and FB influences on layer $2 / 3$ pyramidal neurons mainly involve disynaptic connections via PV neurons that control the spike outputs to axons and proximal dendrites. Unlike FF input, FB input in addition makes a disynaptic link via CR neurons, which may influence the excitability of distal pyramidal cell dendrites in layer 1 .
\end{abstract}

Key words: connection; cortex; GABAergic; visual; calretinin; somatostatin

\section{Introduction}

Rat visual cortex consists of multiple areas that are linked by feedforward (FF) and feedback (FB) connections to an areal hierarchy in which sensory information is represented at multiple levels (Coogan and Burkhalter, 1993). Visual responses in higher areas of monkey visual cortex are completely dependent on FF inputs from lower areas (Schiller and Malpeli, 1977; Girard and Bullier, 1989; Collins et al., 2003). In contrast, FB connections from higher areas of monkey visual cortex modulate the strength of responses in lower areas and contribute to their context, experience, and task dependency (Hupé et al., 1998; Das and Gilbert, 1999; Crist et al., 2001; Lee et al., 2002). Inactivation of FB inputs in monkey has shown that they have enhancing and suppressive influences (Sandell and Schiller, 1982; Hupé et al., 1998; Hupé et al., 2001). These observations suggest that excitation in FF and FB pathways is controlled by circuit-specific inhibition. Because FF and FB connections terminate in different layers (Coogan and Burkhalter, 1993), this may be achieved by the effects of layerspecific inputs to different types of inhibitory neurons.

FF and FB connections are formed by axons of pyramidal cells

Received May 28, 2003; revised Sept. 24, 2003; accepted Sept. 26, 2003.

This work was supported by National Institutes of Health Grants EY-05935, NS3067, and HFSP123/200-B. We thank Katia Valkova for her excellent technical assistance.

Correspondence should be addressed to Dr. Andreas Burkhalter, Department of Anatomy and Neurobiology, 8101, Washington University School of Medicine, 660 South Euclid Avenue, St. Louis, M0 63110. E-mail: burkhala@pcg.wustl.edu.

Copyright $\odot 2003$ Society for Neuroscience $\quad 0270-6474 / 03 / 2310904-09 \$ 15.00 / 0$
(Johnson and Burkhalter, 1997). Most of these form synapses with pyramidal neurons, but $10-20 \%$ of FF and FB inputs are onto GABAergic nonpyramidal cells (Lowenstein and Somogyi, 1991; Johnson and Burkhalter, 1996; Anderson at al., 1998). Although at this level, the organization of FF and FB connections appears similar, recordings of layer $2 / 3$ pyramidal neurons in rat visual cortex show that stimulation of FF inputs elicits excitatory responses that are followed by stronger inhibition than activation of FB inputs (Shao and Burkhalter, 1996). This asymmetry may arise from circuit-specific connections with different types of GABAergic neurons, which provide input to layer $2 / 3$ pyramidal cells.

Neocortical GABAergic neurons are anatomically, physiologically, and chemically diverse (Kawaguchi and Kubota, 1997; Gupta et al., 2000; DeFelipe, 2001; Wang et al., 2002). In rat visual cortex, they constitute at least three distinct families that are distinguished by the expression of parvalbumin (PV), calretinin (CR), and somatostatin (SOM) (Gonchar and Burkhalter, 1997). $\mathrm{PV}$ neurons in layer $2 / 3$ of rat visual cortex are innervated by both FF and FB axons (Gonchar and Burkhalter, 1999a). However, PV neurons account for only $\sim 37 \%$ of GABAergic neurons in layer 2/3 (Gonchar and Burkhalter, 1997). Moreover, dendrites of layer $2 / 3$ pyramidal neurons reach up to layer 1 , which, except for a few dendrites lacks PV (Gonchar and Burkhalter, 1997) and receives stronger FB than FF input (Coogan and Burkhalter, 1993). This raises the question whether CR somata and SOM dendrites, which are abundant in layer 1 (Gonchar and Burkhal- 
ter, 1997), are contacted by FF and FB axons. Thus, we combined tracing of FF and FB connections between area 17 and the higher lateromedial area (LM) with immunolabeling for CR and SOM and used confocal and electron microscopy to determine the relative numerical strengths and pathway specificities of inputs to $\mathrm{CR}$ and SOM neurons in layers 1 and $2 / 3$ of rat visual cortex.

\section{Materials and Methods}

Twenty-one 6- to 8-week-old Long-Evans rats were used in this study. All experimental protocols were approved by the Animals Studies Committee (Washington University, St. Louis, MO) and were in compliance with National Institutes of Health guidelines. For anterograde axonal tracing of FF and FB pathways between visual cortical areas 17 and LM, animals were anesthetized by intraperitoneal injection of a mixture of ketamine $(87 \mathrm{mg} / \mathrm{kg}$ body weight) and xylazine $(13.4 \mathrm{mg} / \mathrm{kg})$. Postsurgical analgesia was provided by subcutaneous injection of buprenorphine $(0.2 \mathrm{mg} / \mathrm{kg})$. In nine rats, pathway tracing was performed with biocytin [2.5\% in 0.01 m phosphate buffer (PB); Sigma, St. Louis, MO ] and in 12 animals with biotinylated dextran amine $(10 \% \mathrm{BDA}$ in $0.1 \mathrm{M} \mathrm{PB}$; Molecular Probes, Eugene, OR). Tracer injections $(\sim 0.02-0.05 \mu \mathrm{l})$ were made by applying pulses of pressurized air to the back of glass pipettes (tip diameter, $\sim 20 \mu \mathrm{m}$ ). Area 17 injections were made at $3.5 \mathrm{~mm}$ lateral $/ 0.5$ $\mathrm{mm}$ anterior to the lambda point, and area LM was injected at $5 \mathrm{~mm}$ lateral $/ 0.5 \mathrm{~mm}$ anterior to lambda. All injections were centered $0.4 \mathrm{~mm}$ below the pial surface.

Visualization of BDA and biocytin for nonfluorescence microscopy. After $18-24 \mathrm{hr}$ of survival, animals were reanesthetized with sodium pentobarbital ( $80 \mathrm{mg} / \mathrm{kg}$ body weight, i.p.) and perfused through the aorta with PBS containing heparin $(100 \mathrm{U} / \mathrm{ml})$, followed by a mixture of paraformaldehyde (4\%), glutaraldehyde $(0.5 \%)$, and picric acid $(0.1 \%)$ in $0.1 \mathrm{M}$ $\mathrm{PB}, \mathrm{pH}$ 7.4. Brains were stored in the same fixative for $2 \mathrm{hr}$ at $4^{\circ} \mathrm{C}$, washed in PBS, and sectioned at $25 \mu \mathrm{m}$ on a Vibratome. Every fifth section was treated with sodium borohydride (1\% in PBS) and incubated in avidinbiotinylated HRP (ABC Elite kit; Vector Laboratories, Burlingame, CA). The HRP reaction was performed in the presence of $3,3^{\prime}$-diaminobenzidine tetrahydrochloride (DAB; $0.05 \%)$ and $\mathrm{H}_{2} \mathrm{O}_{2}(0.005 \%)$. Stained sections were mounted on glass slides, coverslipped, and examined under the light microscope for the presence of FB and FF axons in areas 17 and LM, respectively. Selected sections were counterstained with cresyl violet to reveal cortical layers, including the area 17/LM border. Digital images of labeled axonal projection fields were acquired using a Magnafire CCD camera (Optronics, Goleta, CA).

Visualization of BDA and biocytin combined with $P V, C R, S O M$, and SV2 immunofluorescence. Three series of sections adjacent to those in which nonfluorescence microscopy revealed terminal fields of FF and FB connections were treated with sodium borohydride (1\% in PBS) and incubated for 3-4 hr in avidin—neutralite-Texas Red (1:400; Molecular Probes). The sections were mounted in PBS and viewed under a fluorescence microscope equipped with rhodamine optics. Sections, which contained FF and FB projections, were selected for immunostaining with antibodies against the calcium-binding proteins $\mathrm{PV}$ and CR, the peptide SOM, and the synaptic vesicle protein SV2 (Feany et al., 1992). For simultaneous detection of PV and SOM, goat anti-PV antibodies (1:1000; Swant, Bellinzona, Switzerland) were combined with rabbit anti-SOM antibodies (1:1000) obtained either from Peninsula Laboratories (San Carlos, CA) or as a gift from Dr. R. Benoit (McGill University, Montreal, Canada). Double labeling for CR and SOM was performed with mouse anti-CR (1:1000; Swant) and rabbit anti-SOM antibodies. To check for the presence of synaptic vesicles in boutons of FF and $\mathrm{FB}$ axons that form putative synaptic contacts with SOM and CR neurons, mouse anti-SV2 antibodies (1:100; Developmental Studies Hybridoma Bank, University of Iowa, Iowa City, IA) were used in combination with rabbit anti-SOM or rabbit anti-CR antibodies. Incubations in primary antibodies (overnight, $\left.4^{\circ} \mathrm{C}\right)$ were followed by treatments $\left(2 \mathrm{hr}, 21^{\circ} \mathrm{C}\right)$ with secondary donkey anti-rabbit Cy2 (1:200; Jackson ImmunoResearch Laboratories, West Grove, PA), donkey anti-mouse Cy5 (1:500; Jackson), and donkey anti-goat Cy5 (Jackson). Stained sections were mounted on glass slides, air-dried, dehydrated in ethanol, cleared in citrus-based solvent (Ste- phens Scientific, Riverdale, NJ), and coverslipped in Krystalon (Harleco, Gibbstown, NJ).

Confocal microscopy. A multiphoton confocal microscope (MRC-1024 ES; Bio-Rad, Hercules, CA) was used to study putative synaptic contacts of FF and FB axons with immunolabeled PV, SOM, and CR neurons. The green (Cy2), red (Texas Red), and infrared (Cy5) fluorochromes were excited with, respectively, 488, 568, and $647 \mathrm{~nm}$ emission lines of an argon-krypton laser. Appropriate barrier filters were used to split the emitted light into three distinct channels that were detected by separate photomultipliers. Digitized images were displayed on the screen in three different windows and were superimposed in the fourth window. The laminar position of labeled neurons was studied with a $10 \times$ objective in stacks of 10-40 images (optical thickness $1 \mu \mathrm{m}$ each). Threedimensional (3-D) reconstructions of putative axonal contacts with immunolabeled neurons were performed from stacks of $10-20$ images $(0.5$ $\mu \mathrm{m}$ optical thickness each) acquired with a $60 \times$ objective ( 1.4 numerical aperture). Measurements of distances, areas, and volumes were performed using Laser Sharp and Confocal Assistant software (Bio-Rad).

Electron microscopic identification of FF and FB inputs to $P V, C R$, and SOM neurons. The remaining sections adjacent to those in which the initial screening revealed terminations of FF and FB connections were processed for electron microscopy. Anterogradely transported biocytin and BDA was visualized with avidin and biotinylated HRP (ABC Elite kit; Vector) followed by an HRP reaction in the presence of 3,3',5,5' teramethylbenzidine (TMB; $0.005 \%$ dissolved in $100 \%$ ethanol), ammonium paratungstate (5\%), $\mathrm{NH}_{4} \mathrm{Cl}(0.004 \%)$, and $\mathrm{H}_{2} \mathrm{O}_{2}(0.005 \%)$ in $\mathrm{PB}(0.1 \mathrm{M}$, pH 6) (Weinberg and Van Eyck, 1991; Gonchar and Burkhalter, 1999a). For additional stabilization of the TMB reaction product, sections were further treated with $\mathrm{DAB}(0.05 \%), \mathrm{NH}_{4} \mathrm{Cl}(0.004 \%)$, and $\mathrm{H}_{2} \mathrm{O}_{2}(0.005 \%)$ in $\mathrm{PB}(0.1 \mathrm{M}, \mathrm{pH} 6)$. The reaction was terminated by washing the sections in $\mathrm{PB}(0.1 \mathrm{M}, \mathrm{pH}$ 7.4). The sections were then treated with avidin-biotin blocking solution (Vector), permeabilized in ethanol (60\%), treated with fish gelatin (10\%), and incubated overnight in rabbit anti-CR (Swant; 1:2000) or rabbit anti-SOM antibody (Peninsula; $1: 1000$ ) at $4^{\circ} \mathrm{C}$. The next day, sections were washed in $\mathrm{PB}(0.1 \mathrm{M}, \mathrm{pH} 7.4)$, treated in fish gelatin $(2 \%)$, incubated in biotinylated goat anti-rabbit IgG (Vector; 1:200), transferred into avidin followed by biotinylated HRP and an HRP reaction in the presence of $\mathrm{DAB}$. After several rinses in $\mathrm{PB}$, sections were additionally fixed in $\mathrm{OsO}_{4}(1 \%)$. Sections were dehydrated in ethanol and infiltrated with propylenoxide, which was gradually replaced with Durcupan resin (Fluka, Ronkonkoma, NY). Sections were flatembedded on silicon-coated slides and polymerized at $60^{\circ} \mathrm{C}$ for $48 \mathrm{hr}$. The projection fields of FF and FB connections were identified under the light microscope, and samples containing layers 1 and $2 / 3$ were removed and glued onto blocks of resin. The specimens were resectioned at $50 \mathrm{~nm}$ on an ultramicrotome (Reichert Ultracut E). To provide for systematic, random sampling, alternating series of 10 consecutive thin and 4 semithin $(0.5 \mu \mathrm{m})$ sections were collected. Thin sections were mounted onto 200 mesh nickel grids and stained with uranyl acetate (1\%) followed by Reynolds lead citrate.

Postembedding staining for GABA. Thin sections of SOM or CR immunostained tissue were mounted on 300 mesh nickel grids and stained on drops in a humidified chamber. After a brief rinse in Tris-buffered saline (TBS; $\mathrm{pH} 7.6)$, sections were treated in fish gelatin (2\%), bovine serum albumin (BSA; $1 \%$ ) and Triton X-100 (0.02\% TX-100 in TBS). This was followed by incubation in rabbit anti-GABA antibody (Sigma; 1:5000) diluted in fish gel $(0.2 \%)$, BSA $(0.1 \%)$, and $0.02 \%$ TX-100. The next day, sections were washed in TBS first at $\mathrm{pH} 7.6$ then at $\mathrm{pH} 8.2$ and later incubated for 4-6 hr in goat anti-rabbit IgG conjugated to $15 \mathrm{~nm}$ gold particles (Amersham, Arlington Heights, IL; 1:25 in TBS, pH 8.2). Finally, sections were stained with uranyl acetate and lead citrate as described above.

Quantitative analysis. The numerical density of CR and SOM neurons was determined using the optical disector method (West et al., 1991) as described previously (Gonchar and Burkhalter, 1997). The proportions of anterogradely labeled FF and FB axon terminals that formed synapses with SOM- and CR-labeled profiles in layers $1-2 / 3$ were estimated using both confocal and electron microscopy. For this purpose we randomly selected stacks of confocal images from the termination fields of FF and 
FB connections and counted all boutons formed by interareal axons (100\%), including those that formed putative synaptic contacts with immunolabeled targets. Putative synaptic contacts were counted if: (1) boutons and immunolabeled targets were in the same focal plane and (2) 3-D images (Laser Sharp and Confocal Assistant software; Bio-Rad) viewed at different angles showed no detectable gap between the presynaptic bouton and the postsynaptic target. For movies of confocal images see, http://thalamus.wustl.edu/burkhalterimages/.

In the electron microscope, thin sections in which both immunostaining and tracer labeling appeared complete were scanned, and all synapses formed by anterogradely labeled axons were photographed at $20,000 \times$. BDA or biocytin-labeled axon terminals were identified by the presence of rod-shaped TMB reaction product. Symmetric and asymmetric synapses were distinguished based on morphological criteria described by Peters and Palay (1996). Immunolabeled SOM and CR profiles were filled with amorphous DAB reaction product. To estimate synaptic inputs to SOM and CR neurons in the neuropil, pictures $(10,000 \times)$ were taken in systematically randomly selected sections. Negatives were enlarged 2.5 times, and synapses were counted on prints. The proportion of synapses with SOM and CR profiles were expressed relative to the total number of FF and FB synapses. To assess whether FF and FB connections selectively target different types of interneurons, we compared the proportions of FF and FB inputs to SOM and CR profiles in a given pathway with the proportions of all asymmetric synapses onto SOM and CR neurons in the same region. The areas, perimeters, and diameters of axon terminals were measured on prints using digitizing tablet and NIH Image software. Statistical analyses were performed using the paired Student's $t$ test. $P$ values of $<0.05$ indicated significant differences.

Preparations of figures. Electron photomicrographs were printed from negatives by using Eastman Kodak (Rochester, NY) polycontrast paper, or were produced by scanning negatives on a flatbed scanner at $1200 \mathrm{dpi}$ resolution. Adobe Photoshop software (Adobe Systems, San Jose, CA) was used to make linear adjustments of brightness and contrast of both confocal and electron microscopic images.

\section{Results}

\section{Distribution and morphology of CR and SOM neurons}

Antibodies against CR stain neurons across all layers of areas 17 and LM (Fig. 1A,B). GABA immunogold labeling of CRimmunostained neurons shows that these cells are GABAergic (see Fig. 7B) (Gonchar and Burkhalter, 1999b). Triple immunolabeling further demonstrates that PV and SOM are not expressed in CR neurons (see Fig. 3A), suggesting that CR neurons represent a distinct family of GABAergic neurons (Gonchar and Burkhalter, 1997).

The laminar distribution of CR neurons is similar in areas 17 and LM: $\sim 75 \%$ of neurons reside in layers $1-4$, and the remainder is distributed across layers 5 and 6 (Table 1). In both areas, layer 1 contains $\sim 16-18 \%$ of CR neurons (Table 1 ). Of the three cell types studied here, only CR neurons have somata in layer 1 (Gonchar and Burkhalter, 1997).
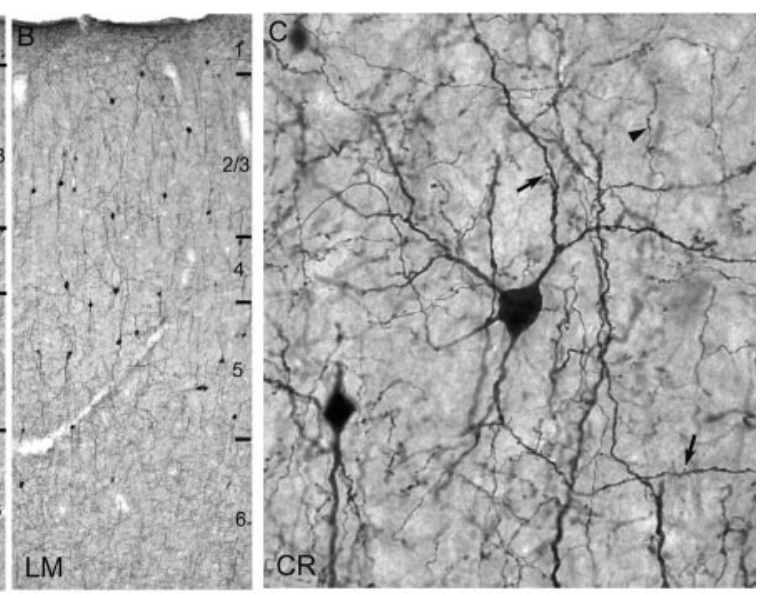

Figure 1. CR-and SOM-immunolabeled neurons in rat visual cortex. $A, B$, Laminar distribution of CR neurons in area 17 and the secondary visual area, LM. Most CR neurons are located in superficial layers. Layer 1 contains CR somata, but lacks SOM $(D, E)$ and V (Fig. 2C) cell bodies. C, CR stains cell bodies including the entire dendritic tree. Dendritic spines (arrows) are rare. The neuropi immunolabeling reveals soma, primary, and secondary dendrites. The neuropil contains labeled axons and boutons (arrowheads).

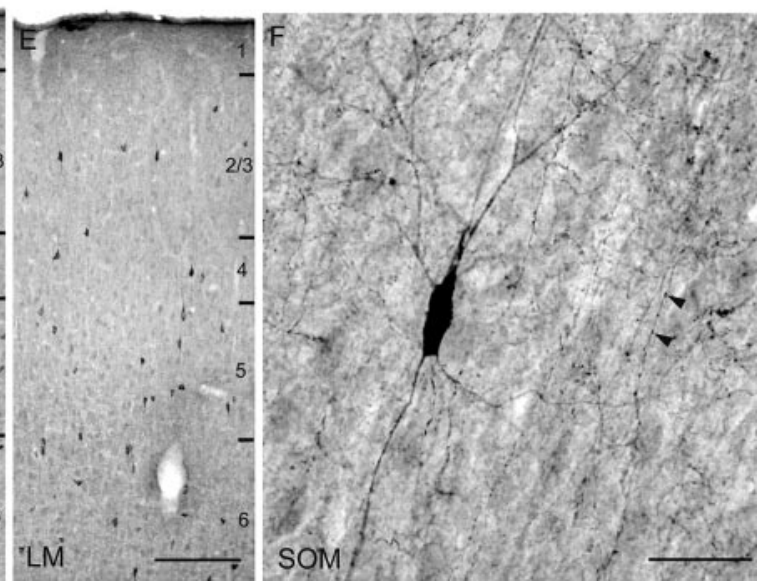

Scale bars: (in $E) A, B, D, E, 100 \mu \mathrm{m}$; (in F), C, F, $20 \mu \mathrm{m}$.

CR immunoperoxidase and immunofluorescence labels somata, dendrites, and axons in Golgi-like manner (Figs. $1 B, C$, $2 A, B$ ). Most CR neurons have oval cell bodies (short axis 8-12 $\mu \mathrm{m}$, long axis $10-15 \mu \mathrm{m}$ ) and dendritic trees with bipolar or double bouquet morphologies (Figs. 1C, 2B, 3E). Dendritic spines are rare (Fig. $1 C$ ). In layers $2-6$ most CR axons are vertically oriented, whereas in layer 1 they run horizontally.

SOM staining is present in a distinct population of nonpyramidal neurons that lacks PV and CR (Fig. $3 A$ ) but contains GABA (see Figs. 6C, $7 A, C, D$ ). In both areas 17 and LM, SOM-labeled somata are scattered throughout layers $2-6$, but they are absent from layer 1 (Fig. 1D,E). Unlike CR neurons, which are more abundant in superficial layers, SOM neurons are more frequent in deep layers (Table 1). The numerical density of SOM neurons in layer 5 is significantly ( $p<0.05, t$ test) higher in LM than in area 17 (Table 1), but no areal differences were found in the remaining layers (Table 1). SOM neurons are clustered in small groups from which CR neurons are often excluded. Although the horizontal distribution of SOM neurons appears nonuniform, there is no obvious orderly pattern.

SOM neurons are aspiny nonpyramidal cells with multipolar, bitufted, or bipolar dendritic trees. SOM immunofluorescence and immunoperoxidase stainings often reveal large portions of the dendritic tree (Fig. $1 F$ ). However, SOM staining is incom- 
Table 1. CR and SOM neurons in areas 17 and LM of rat visual cortex

\begin{tabular}{|c|c|c|c|c|c|c|c|c|}
\hline \multirow[b]{3}{*}{ Layer } & \multicolumn{4}{|l|}{ Area 17} & \multicolumn{4}{|l|}{ Area LM } \\
\hline & \multicolumn{2}{|l|}{$C R$} & \multicolumn{2}{|l|}{ SOM } & \multicolumn{2}{|l|}{$C R$} & \multicolumn{2}{|l|}{ SOM } \\
\hline & Density & $\%$ & Density & $\%$ & Density & $\%$ & Density & $\%$ \\
\hline 1 & $1484 \pm 288$ & 18.1 & 0 & 0 & $1527 \pm 304$ & 16.1 & 0 & 0 \\
\hline $2 / 3$ & $2501 \pm 294^{*}$ & 30.5 & $2105 \pm 348$ & 21.1 & $3080 \pm 268^{*}$ & 32.6 & $2038 \pm 265$ & 21.8 \\
\hline 4 & $2106 \pm 198$ & 25.7 & $2768 \pm 381$ & 27.6 & $2410 \pm 212$ & 25.5 & $2301 \pm 356$ & 24.6 \\
\hline 5 & $994 \pm 222$ & 12.1 & $3095 \pm 278^{\#}$ & 30.9 & $1275 \pm 227$ & 13.5 & $2431 \pm 226^{\#}$ & 26 \\
\hline 6 & $1119 \pm 163$ & 13.6 & $2045 \pm 395$ & 20.4 & $1160 \pm 209$ & 12.3 & $2589 \pm 278$ & 27.6 \\
\hline Mean 1-6 & $1425 \pm 168$ & & $2353 \pm 235$ & & $1802 \pm 163$ & & $2227 \pm 189$ & \\
\hline Number under $1 \mathrm{~mm}^{2}$ & $1982 \pm 234^{\wedge}$ & & $3272 \pm 326$ & & $2685 \pm 244^{\wedge}$ & & $3318 \pm 281$ & \\
\hline
\end{tabular}

Numerical densities in $1 \mathrm{~mm}^{3}$ and number of neurons under $1 \mathrm{~mm}^{2}$ of cortical surface are indicated. ${ }^{*, \#, \wedge}$ Significant differences between areas 17 and LM.
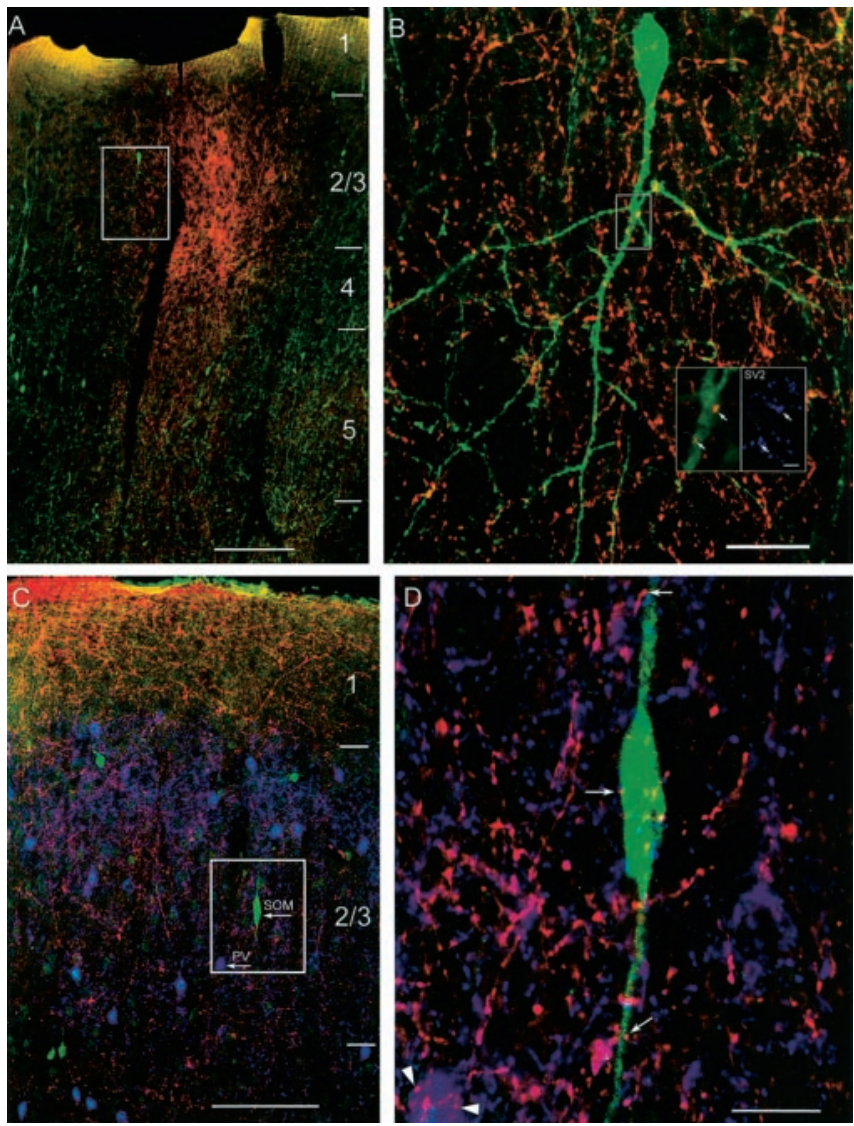

Figure 2. Confocal microscopic images of putative synaptic $F F$ and $F B$ inputs to $C R$ and SOM expressing GABAergic neurons in rat visual cortex. $A$, Anterogradely labeled terminal field of FF axons (red) in area LM after injection of biocytin into area 17. CR-labeled somata and dendrites are shown in green. The boxed area is shown at higher magnification in B. Scale bar, $100 \mu \mathrm{m}$. B, CRimmunolabeled neuron (green) located in the terminal field of FF axons (red) in layer 2/3 of area LM. Insets (bottom right) show two high-power images taken of boxed area in the center of the picture. Arrows indicate putative contacts (see movies athttp://thalamus.wustl.edu/burkhalterimages/) of FF boutons (red) with CR dendrites (green). The colocalization of SV2 (blue, arrows) in axon terminals (red, arrows) indicates that they contain vesicle proteins that are used in synapses. Optical thickness of section: $5 \mu \mathrm{m}$. Scale bars: B, $20 \mu \mathrm{m}$; inset, $2 \mu \mathrm{m}$. C, Anterogradely labeled FB axons (red) in layers 1 and $2 / 3$ of area 17 after injection of biocytin into area LM. PV (blue) and SOM (green) immunolabeled neurons. Boxed area contains SOM and PV neurons (arrows) that are shown at higher magnification in $D$. Scale bar, $100 \mu \mathrm{m}$. D, High-magnification image of boxed area shown in C, indicating putative synaptic FB (red) inputs to SOM (green, arrows) and PV (blue, arrowheads) neurons. Optical thickness of section: $5 \mu \mathrm{m}$. Scale bar, $10 \mu \mathrm{m}$.

plete in many neurons and rarely extends beyond secondary dendritic branches (Figs. 2D, 3D). SOM-stained axons with boutons of passage and terminal swellings run mostly vertically in layer 2-6 and horizontally in layer 1 (Fig. $1 F)$.
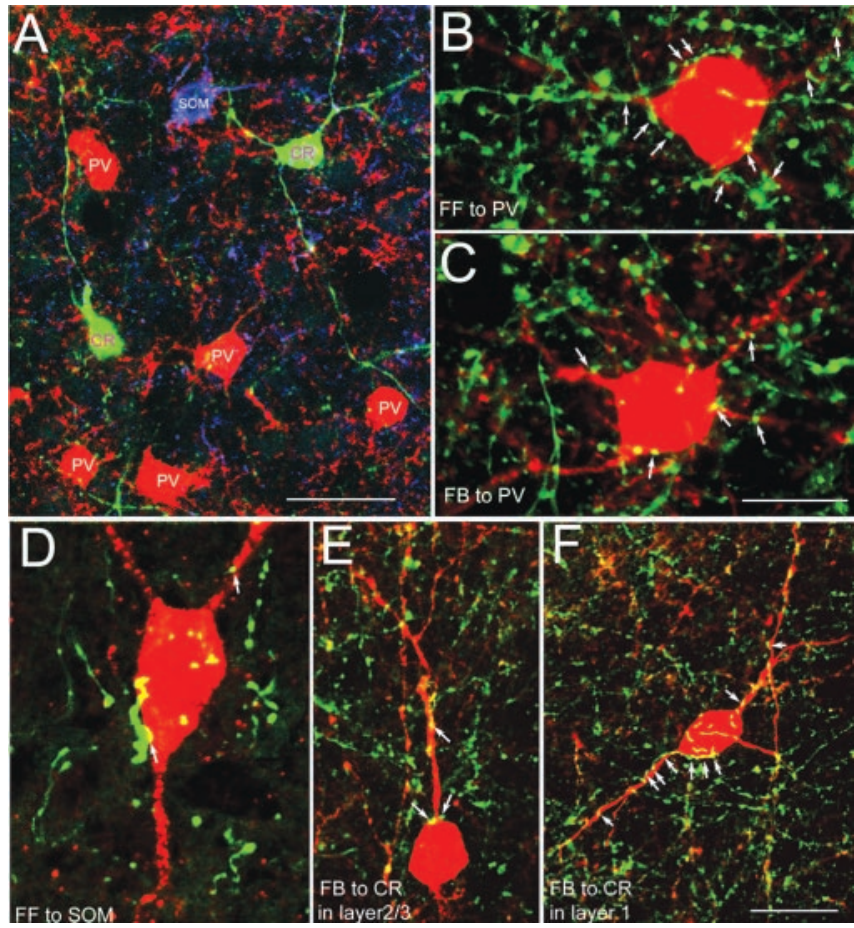

Figure 3. $P V, C R$, and SOM expression in different types of GABAergic neurons that are differentially innervated by FF and $\mathrm{FB}$ axons. $A$, Triple labeling with antibodies against PV (red), CR (green), and SOM (blue) in layer 2/3 of rat area 17 showing that each cell is labeled by only a single marker. Scale bar, $25 \mu \mathrm{m} . B, C$, Confocal images of anterogradely biocytin-labeled FF and $\mathrm{FB}$ axons (green) forming multiple putative synaptic contacts (arrows) with PV neurons (red) in layer 2/3 of areas $L M(B)$ and 17 ( C). Scale bar: (in C) B, C, $10 \mu \mathrm{m}$. D, Biocytin-labeled FF axons (green) making infrequent contacts (arrow) with SOM (red) neuron in layer 2/3 of area LM. $E$, Biocytin-labeled FB axons (green) making occasional contacts (arrows) with CR (red) neuron in layer $2 / 3$ of area $17 . F, F B$ axons (green) in layer 1 of area 17 strongly innervate $C R$ (red) neurons. Arrows indicate putative synaptic contacts. Scale bar: (in $F$ ) $D-F, 10 \mu \mathrm{m}$.

\section{Confocal microscopy}

Injections of biocytin or $\mathrm{BDA}$ into area 17 anterogradely label $\mathrm{FF}$ axons that terminate in area LM (Fig. $2 A, C$ ). Both tracers are confined mainly to axons and boutons, and retrogradely labeled cells are rare (Fig. $2 A, B$ ). The density of FF axon terminals is densest in layers $2 / 3$ and 4 , intermediate in layers 5 and 6 , and extremely low in layer 1 (Fig. $2 \mathrm{~A}$ ).

Tracer injections into area LM label FB axons in area 17. Unlike FF connections, $\mathrm{FB}$ input to layer 1 is very strong (Fig. $2 \mathrm{C}$ ). $\mathrm{FB}$ connections to layers $2 / 3,5$, and 6 are less dense than to layer 1 , and input to layer 4 is extremely sparse (Fig. 2C). Similar to FF connections (Fig. 2B), FB axons form boutons of passage and terminal swellings (Fig. 2D). The laminar distributions of con- 
A
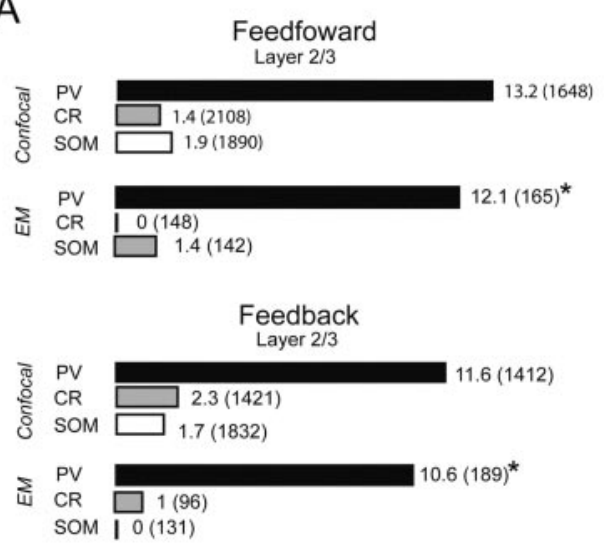

B

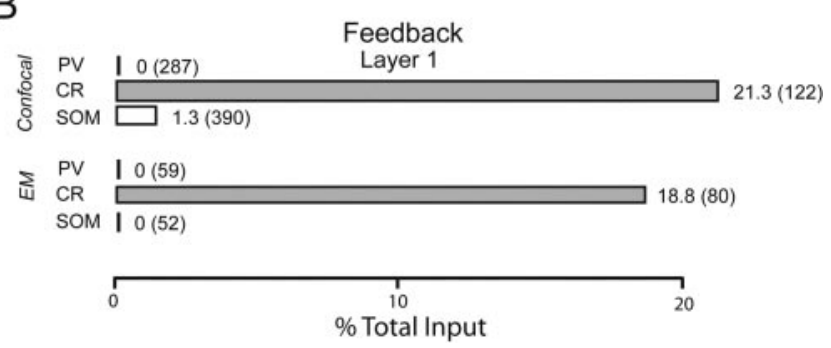

Figure 4. Relative strengths of $F F$ and $F B$ inputs to $P V, C R$, and SOM neurons in rat visual cortex, assessed by confocal and electron microscopy (EM). A, Percentage of all $\mathrm{FF}$ and $\mathrm{FB}$ connections providing inputs to $\mathrm{PV}, \mathrm{CR}$, and $\mathrm{SOM}$ neurons in layer 2/3. Total number of boutons and synapses is indicated in parentheses. Asterisks indicate EM data from Gonchar and Burkhalter (1999a). B, Percentage of all FB connections making inputs to PV, CR, and SOM cell bodies and dendrites in layer 1 .

nections observed with fluorescence microscopy are similar to those seen with nonfluorescent markers (Johnson and Burkhalter, 1997; Gonchar and Burkhalter, 1999a).

\section{Putative FF and FB inputs to $\mathrm{CR}$ neurons}

In layer $2 / 3$ of areas 17 and $\mathrm{LM}$, close appositions of FF and FB boutons with CR neurons are rare (Figs. $2 A, 3 E$ ). This is true even for neurons that lie in the core of $\mathrm{FF}$ and $\mathrm{FB}$ projections and whose dendritic trees are completely labeled. Many boutons at putative synaptic appositions colocalize SV2, which indicates the presence of synaptic vesicles (Fig. $2 B$, inset). Quantitative analyses confirm the qualitative impression and show that of all $\mathrm{FF}$ and FB boutons contained in the dendritic field of CR neurons in layer $2 / 3$, only $\sim 1-2 \%$ form putative synapses onto $C R$ dendrites (Fig. 4A). The virtual absence of FF and FB inputs to layer $2 / 3 \mathrm{CR}$ neurons contrasts with the dense innervation of layer $2 / 3 \mathrm{PV}$ neurons by $\mathrm{FF}$ and $\mathrm{FB}$ axons (Figs. $2 D, 3 B, C$ ).

Unlike FB inputs to layer 2/3 CR neurons (Fig. 3E), FB inputs to $\mathrm{CR}$ neurons in layer 1 are remarkably dense and mainly onto somata and proximal dendrites (Fig. $3 F$ ). Quantitative analyses show that of all FB axon terminals in layer 1, FB inputs to CR neurons account for $21.3 \%$ (Fig. $4 B$ ). This proportion is almost an order of magnitude higher than of $\mathrm{FB}$ inputs to $\mathrm{CR}$ neurons in layer $2 / 3$ (Fig. $4 A$ ).

Given the sparse FF input to layer 1 (Fig. 2A), it is not surprising that FF inputs to CR neurons is essentially lacking. Despite intense scrutiny, we have only found a single putative FF input, which precluded quantitative analysis.

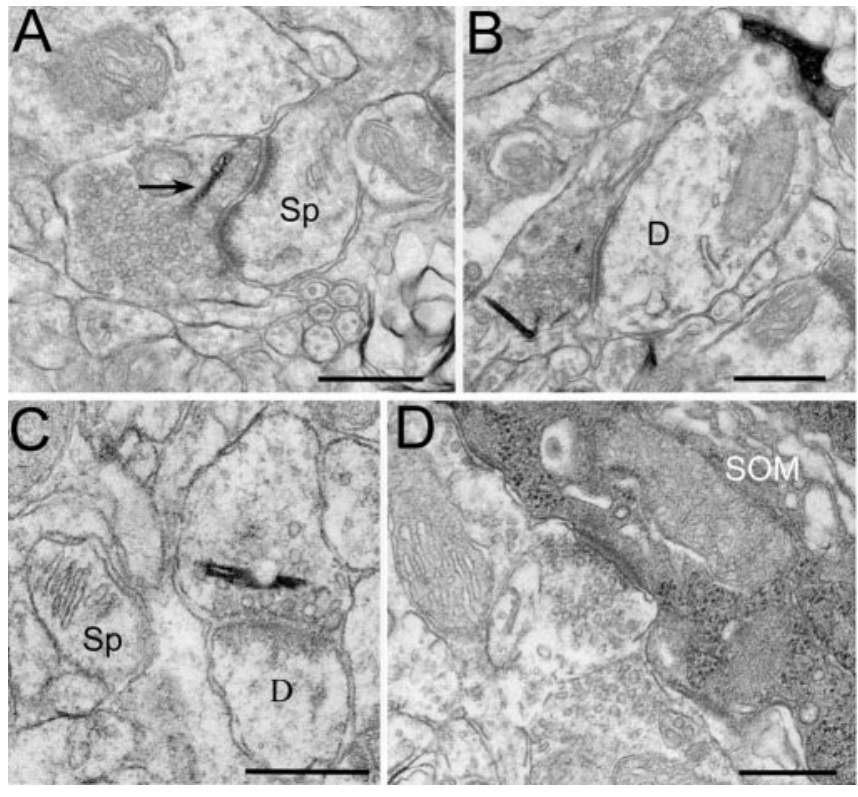

Figure 5. Synaptic targets of $\mathrm{FF}$ and $\mathrm{FB}$ connections in rat visual cortex. A, Anterogradely biocytin-labeled (needle-shaped TMB/tungstate reaction product indicated by arrow) FF axon terminal in layer $2 / 3$ of area LM. The large axon terminal contains round vesicles and forms asymmetric synapse with dendritic spine (Sp), which contains a prominent spine apparatus. $B$, Large biocytin-labeled (see dark TMB/tungstate crystal on lower left) FF terminal forming asymmetric synapse with dendritic shaft (D).C, Biocytin-labeled (see dark TMB/tungstate crystal) FB axon terminal forming asymmetric synapse with small dendrite $(D)$. Nearby spine shows prominent spine apparatus (Sp). D, Asymmetric synapse of SOM-immunoreactive soma (SOM, indicated by dark $D A B$ reaction product). Scale bars: $A-D, 0.25 \mu \mathrm{m}$.

\section{Putative FF and FB inputs to SOM neurons}

$\mathrm{FF}$ and $\mathrm{FB}$ axons in layer $2 / 3$ of areas 17 and LM form extremely few contacts with SOM neurons. All of these putative FF and FB synapses were onto somata and proximal dendrites (Figs. $2 D$, $3 D$ ). Of all FF and FB boutons in layer $2 / 3$ only $\sim 2 \%$ form putative synapses with SOM neurons (Fig. 4A).

\section{Putative FF and FB inputs to PV neurons}

The sparse FF and FB inputs to CR and SOM neurons contrasts with the dense innervation of PV somata and dendrites in both pathways (Fig. 3B,C). Of all boutons encountered in the projection zone, $\sim 13 \%$ of $\mathrm{FF}$ and $\sim 12 \%$ of $\mathrm{FB}$ axon terminals form putative contacts with layer 2/3 PV neurons (Fig. 4A). All efforts to find putative FF and FB contacts with PV dendrites in layer 1 were unsuccessful.

\section{Electron microscopy}

\section{Synaptic targets of FF and FB terminals}

In the electron microscope, anterogradely BDA- or biocytinlabeled FF and FB boutons are easily identified by the presence of needle-shaped TMB crystals (Figs. $5 A-C, 7 E, 8 A-C$ ). The vast majority of boutons formed by FF $(98.7 \% ; 462$ of 468$)$ and FB axons (99\%; 394 of 398) contain large (35-40 nm in diameter) round vesicles. In the remaining $\sim 1 \%$ of $\mathrm{FF}$ and $\mathrm{FB}$ boutons, vesicles are small and elongated (short axis $30 \mathrm{~nm}$, long axis 40 $\mathrm{nm})$. Of all $\mathrm{FF}(n=290)$ and $\mathrm{FB}(n=211)$ boutons in which we found synaptic contacts, $\sim 99 \%$ of synapses are asymmetric. Most of these synapses in the FF $(80.6 \% ; 234$ of 290$)$ and FB $(84.8 \%$; 179 of 211) pathways are onto spines and thin dendrites (Fig. $5 A, C)$. The remaining inputs are onto dendritic shafts and cell bodies (Figs. 5B,D). 


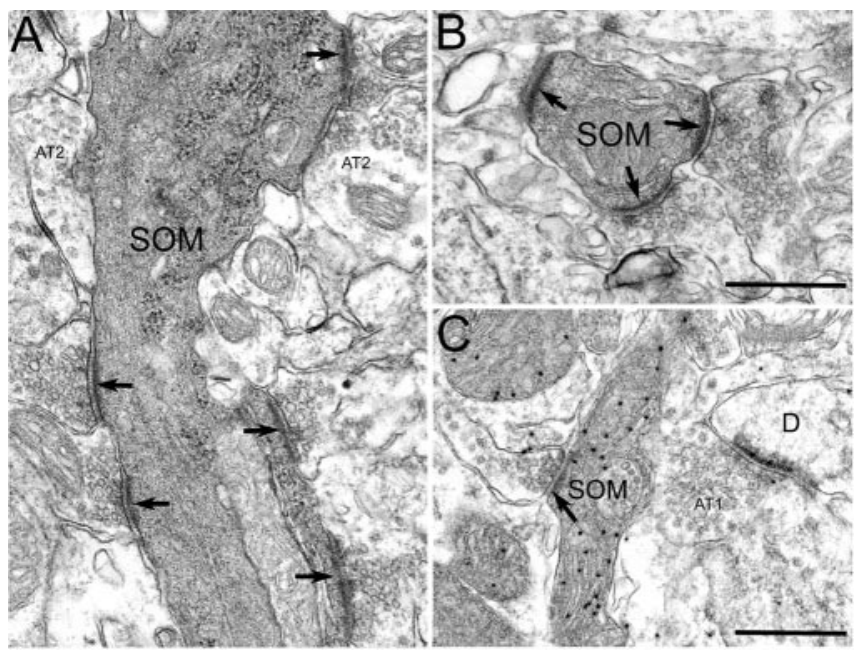

Figure 6. Asymmetric and symmetric synaptic inputs to SOM neurons in layer $2 / 3$ of rat area 17. A, Densely innervated SOM-immunoreactive (dark amorphous DAB reaction product) dendrite with multiple inputs from asymmetric (arrows) and symmetric Gray type 2 synapses (type 2 axon terminal, AT2). B, Small SOM dendrite with inputs from three asymmetric synapses. Scale bar, $0.25 \mu \mathrm{m}$. C, GABA immunogold-labeled (black dots), SOM-immunoreactive dendrite with input from asymmetric synapse (arrow). Nearby GABA-negative dendrite (D) receives inputs from asymmetric, Gray type 1 synapse (type 1 axon terminal, AT1). Scale bar, $0.5 \mu \mathrm{m}$.

\section{Unlabeled inputs to CR and SOM neurons}

CR- and SOM-immunoreactive somatic, dendritic, and axonal profiles are identified by the content of amorphous $\mathrm{DAB}$ reaction product (Figs. 5D, 6A-C, 7A-E, 8A,B). CR- and SOM-labeled somata are often contacted by asymmetric and symmetric synapses (Figs. $5 D, 6 A, 7 A, B$ ), which is a characteristic feature of nonpyramidal neurons (Peters et al., 1991). Postembedding immunogold staining with antibodies against GABA shows that all immunoperoxidase-labeled CR (41 of 41) and SOM (37 of 37) somata, dendrites, and axon terminals are GABAergic (Figs. $6 C$, $7 A-D)$. CR somata and dendrites consistently show smooth plasma membranes (Fig. 5B), whereas SOM neurons have scalloped outlines (Figs. 5D, 6A, 7C). CR neurons always contain more mitochondria than SOM neurons.

CR and SOM neurons in layer 2/3 of areas 17 and LM receive numerous symmetric and asymmetric synaptic inputs. Asymmetric synapses in layer $2 / 3$ of area 17 account for $80.2 \%$ (1038 of 1294) of all synaptic profiles. A similar proportion (79.8\%; 1011 of 1267) of asymmetric synapses was found in layer $2 / 3$ of area LM.

Most ( $\sim 98 \%$ ) asymmetric synaptic inputs to CR neurons are onto dendrites, and very few are onto somata. These inputs account for $6.3 \%$ ( 56 of 885 ) of all asymmetric synapses in layer $2 / 3$ of area 17 . A similar percentage $(6.5 \% ; 31$ of 477$)$ of asymmetric inputs was found to CR neurons in layer $2 / 3$ of LM.

SOM neurons receive fewer asymmetric synapses than CR neurons. They account for only $1.8 \%$ (11 of 617) of all asymmetric synapses in layer $2 / 3$ of area 17 . Similarly, in layer $2 / 3$ of LM, asymmetric inputs to SOM neurons account for only $1.6 \%$ (8 of $505)$ of all asymmetric synapses. The small proportions of inputs are surprising because many SOM somata and dendrites are strongly innervated (Fig. 6A,B). One possible explanation is that a substantial fraction of inputs are located on distal SOM dendrites (Fig. 6B,C) and that many of these inputs were missed because of incomplete immunolabeling of thin dendrites. Alternatively, the innervation density of SOM neurons may be heterogeneous.
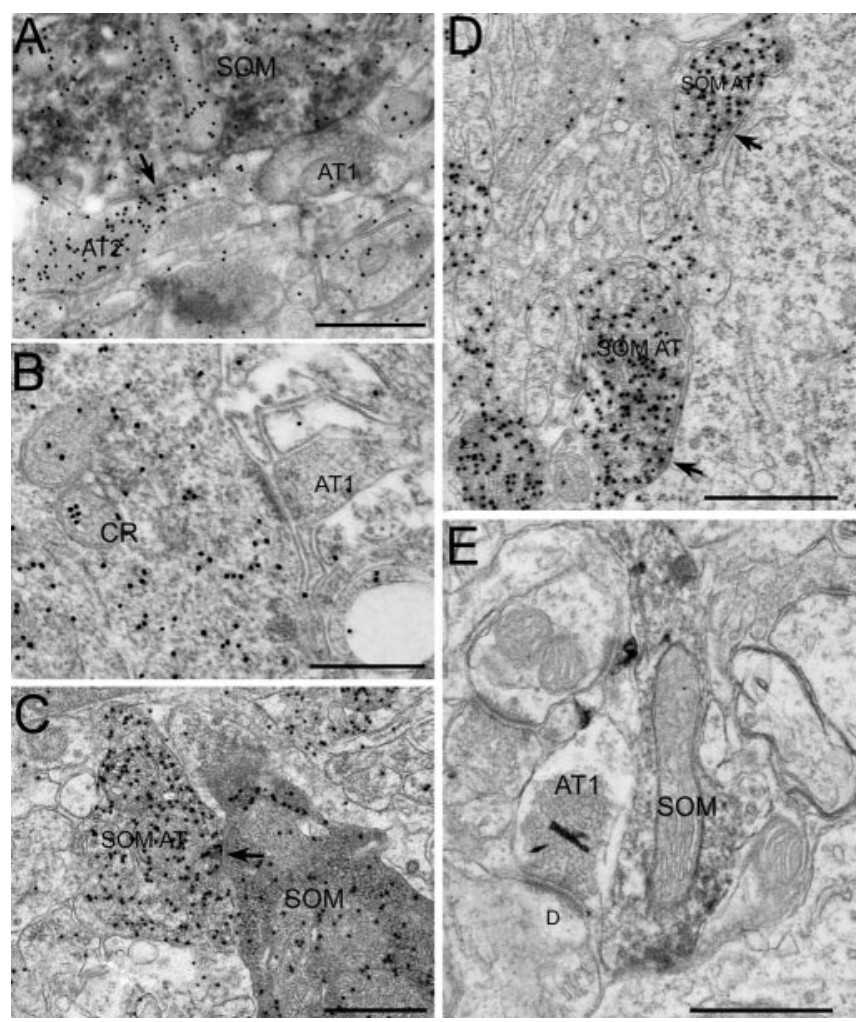

Figure 7. SOM and $C R$ neurons in layer $2 / 3$ of rat visual cortex are GABAergic and receive inputs from GABAergic and non-GABAergic synapses. $A$, Coexpression of SOM (dark amorphous $D A B$ reaction product) and $G A B A$ immunogold particles in cell body, which receives inputs from symmetric GABAergic (AT2, arrow) and asymmetric, GABA-negative synapse (AT1) synapses. B, Coexpression of $C R$ and $G A B A$ in cell body that receives asymmetric synapse from GABA-negative, type 1 axon terminal (AT1). C, Large SOM/GABA double-labeled axon terminal (SOM AT) which forms symmetric synapse (arrow) on SOM/GABA double-labeled cell body. D, SOM-labeled GABAergic axon terminals (SOM AT) forming symmetric synapses (arrows) with SOM-negative/GABA-negative cell body. E, Anterogradely biocytin-labeled (dark TMB/tungstate crystal) FF axon terminal in close association with SOM-immunoreactive dendrite (SOM) forming asymmetric synapse (AT1) with nearby unlabeled dendrite (D). Scale bars: $A-E, 0.5 \mu \mathrm{m}$.

FF and FB inputs to CR and SOM neurons in layer 2/3

In agreement with the confocal microscopic analysis, examination in the electron microscope shows that both FF and FB inputs to CR neurons are sparse. Of a total of 148 asymmetric FF synapses in layer 2/3 of area LM, not a single input was found onto a CR profile (Fig. 4). Similarly, of $96 \mathrm{FB}$ synapses in layer $2 / 3$ of area 17 we observed only a single input to a CR dendrite (Figs. 4, $8 \mathrm{~A}$ ). The paucity of anterogradely labeled FF and FB synapses on $\mathrm{CR}$ neurons contrasts with the large proportion of unlabeled asymmetric inputs to CR neurons (see above).

In SOM-stained tissue, we examined a total of $142 \mathrm{FF}$ synapses in layer 2/3 of LM. Although many of these synapses are surrounded by profiles of SOM somata and dendrites, we found only two $(1.4 \%)$ that formed contacts with SOM elements (Figs. 4, $8 C)$. It is intriguing that in many cases FF axon terminals are closely apposed to SOM dendrites but fail to make contact and instead form synapses with nearby unlabeled profiles (Fig. 7E). FB inputs to SOM neurons in layer $2 / 3$ are similarly sparse, and despite intense scrutiny, we failed to find a single one (Fig. 4).

Inputs to CR neurons in layer 1

Of the three types of GABAergic neurons studied, only CR neurons have cell bodies in layer 1 . Most dendrites of these neurons are confined to layer 1 , and only few short branches descend into layer $2 / 3$. In contrast to the sparse innervation of CR neurons in 


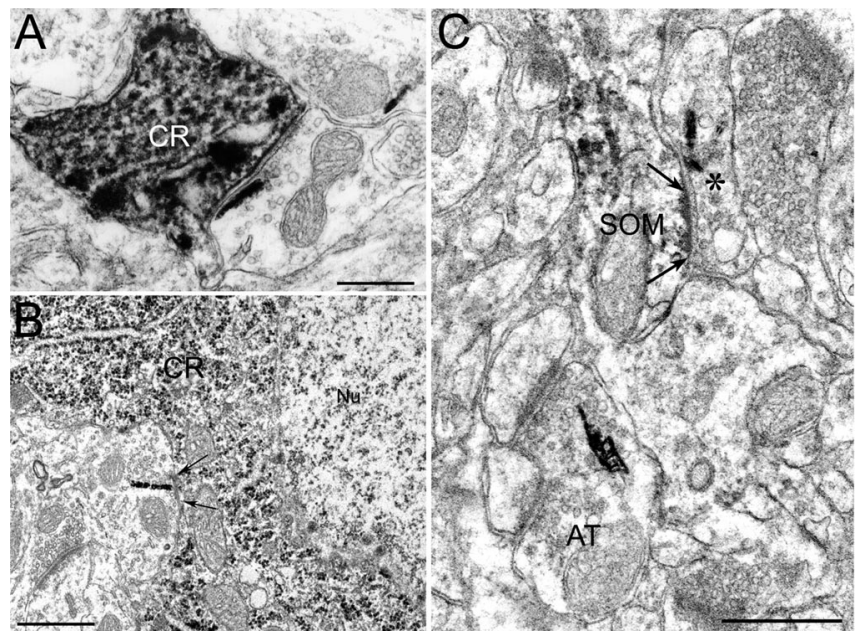

Figure 8. FB inputs to CR and SOM neurons in rat area 17. Anterogradely biocytin-labeled (dark TMB/tungstate crystal) FB axon terminals forming asymmetric synapse with CR dendrite in upper layer 2/3 $(A)$ and with CR immunoreactive cell body in layer 1 ( $B$, arrows). Nu indicates cell nucleus. C, FF input to SOM neuron in rat area LM. Two anterogradely biocytin-labeled (dark TMB/tungstate crystals) FF axon terminals in layer 2/3 of area LM which both form asymmetric synapses. The FF terminal marked with an asterisk makes synaptic contact (arrows) with SOMimmunoreactive dendrite. The terminal marked AT forms symmetric synapse onto unlabeled dendrite. Scale bars: $A, 0.2 \mu \mathrm{m} ; B, C, 0.5 \mu \mathrm{m}$.

layer 2/3, FB inputs to CR neurons in layer 1 of area 17 are much more abundant. All of these synapses are asymmetric and are onto somata and dendrites (Fig. $8 \mathrm{~B}$ ). FB inputs to CR neurons in layer 1 of area 17 account for $21.3 \%$ of the total projection (Fig. 4). This high percentage of FB inputs contrasts with the much smaller proportion of $5.1 \%$ (20 of 395) of unlabeled asymmetric synapses on CR profiles in the neuropil of layer 1 of area 17. CR neurons in layer 1 also receive inputs from symmetric synapses, some of which are CR-immunoreactive.

Unlike FB connections, FF inputs to layer 1 of area LM are extremely sparse. Of a total of 6 FF synapses found in the electron microscope, not a single one forms synaptic contacts with $\mathrm{CR}$ neurons (Fig. 4).

\section{Discussion}

This study demonstrates that FF and FB inputs to CR and SOM neurons in layer $2 / 3$ are sparse and $\sim 5-10$ times weaker than inputs to PV neurons, which are the main GABAergic target of FF and $\mathrm{FB}$ pathways. In contrast, inputs to CR neurons in layer 1 are circuit-specific and only found in the FB pathway. The results suggest that in FF and FB circuits excitatory responses of layer $2 / 3$ pyramidal neurons are shaped by dissimilar sets of GABAergic neurons, which in the FB pathway may include inputs from layer 2/3 PV neurons to somata and proximal dendrites and inputs from CR neurons to dendrites in layer 1 .

\section{Sparse inputs to CR and SOM neurons in layer $2 / 3$}

Most FF and FB connections between lower and higher cortical areas are made by pyramidal neurons that use glutamate as their transmitter (McDonald and Burkhalter, 1993; Johnson and Burkhalter, 1994; Domenici et al., 1995). In monkey, cat and rat most of these connections form asymmetric synapses on spines and dendritic shafts of pyramidal cells (Fisken et al., 1975; Lowenstein and Somogyi, 1991; Johnson and Burkhalter, 1996; Anderson et al., 1998; Anderson and Martin, 2002; this study). The remaining $10-20 \%$ of inputs are to aspiny, GABAergic neu- rons (Lowenstein and Somogyi, 1991; Johnson and Burkhalter, 1996; Anderson et al., 1998; Anderson and Martin, 2002).

In rat cerebral cortex, PV, CR, and SOM mark different GABAergic neurons (Gonchar and Burkhalter, 1997; Kawaguchi and Kubota, 1997). Using triple immunofluorescence labeling, our results demonstrate that neurons expressing one of the three markers do not stain the other two, suggesting that PV, CR, and SOM neurons constitute distinct families.

CR neurons in layer $2 / 3$ of rat visual cortex account for $\sim 20 \%$ of GABAergic neurons (Gonchar and Burkhalter, 1997) or 5\% of all neurons (Beaulieu, 1993; Gonchar and Burkhalter 1997; this study). The numerical density of CR neurons in area LM is $20-26 \%$ higher than in area 17, which parallels the difference found between V1 and V2 in monkey (DeFelipe et al., 1999). CR neurons in layer $2 / 3$ of rat and monkey visual cortex are densely innervated by symmetric and asymmetric synapses (Meskenaite, 1997; Gonchar and Burkhalter, 1999b). Of all asymmetric synapses in layer $2 / 3, \sim 6 \%$ are onto CR neurons. In contrast, of all FF and $\mathrm{FB}$ inputs to layer $2 / 3$ maximally $1 \%$ are to $\mathrm{CR}$ neurons, suggesting that $\mathrm{FF}$ and $\mathrm{FB}$ axons in layer $2 / 3$ selectively avoid $\mathrm{CR}$ neurons. The avoidance of CR neurons by FF and FB axons supports findings that different types of GABAergic cells have different sources of excitatory inputs and that the numerical densities of these inputs are cell type-specific (Gulyás et al., 1999; Dantzker and Callaway, 2000).

SOM neurons account for $\sim 16 \%$ of GABAergic neurons or $\sim 3 \%$ of neurons in layer $2 / 3$ of visual cortex (Beaulieu, 1993; Gonchar and Burkhalter, 1997, this study). We have found that maximally $2 \%$ of $\mathrm{FF}$ and $\mathrm{FB}$ inputs go to SOM neurons in layer $2 / 3$. This proportion is similar to the $\sim 1.8 \%$ of unlabeled asymmetric inputs to SOM neurons. Because immunostaining of SOM dendrites is incomplete, these percentages represent minimal estimates. Assuming that the subcellular distributions of inputs to SOM neurons in neuropil and interareal circuits are similar, it appears that FF and FB axons neither specifically target nor selectively avoid SOM neurons.

\section{PV neurons are main GABAergic target of FF and FB inputs to layer $2 / 3$}

The small percentage of inputs to CR and SOM neurons differs from the large proportion of FF and FB inputs to PV neurons (Fig. 4) (Gonchar and Burkhalter, 1999a). This difference may be attributable to the higher proportion of PV than CR and SOM neurons (Gonchar and Burkhalter, 1997), the large dendritic tree of PV neurons (Wang et al., 2002), and/or a denser innervation of individual PV neurons (Gulyás et al., 1999). The proportion of FF and FB inputs $(11-12 \%)$ to PV neurons is similar to that (9$10 \%$ ) of asymmetric inputs in the neuropil (Gonchar and Burkhalter, 1999a), suggesting that relative to the total input, FF and $\mathrm{FB}$ axons have no particular preference for PV neurons. Importantly, however, FF and FB axons avoid CR and SOM neurons and select PV neurons as their main GABAergic targets. The preference for PV neurons over other types of interneurons argues against an interareal connectivity that is strictly determined by axonal density (Braitenberg and Schüz, 1998; Traczy-Hornoch et al., 1999). PV neurons were shown to be preferred targets in rat thalamocortical pathway (Staiger et al., 1996) and in feedback connections from layer 3 to 4 of cat visual cortex (Thomson et al., 2002). Furthermore, in rat visual cortex putative PV neurons in layer $2 / 3$ were shown to be preferentially targeted by inputs from layer 4 , but receive few inputs from layer 5 (Dantzker and Callaway, 2000). Together these data suggest that the numerical strength of inputs to PV neurons can be circuit-specific and may 
underlie pathway-specific inhibition. However, our findings suggest that PV neurons have similar representations in FF and FB circuits. Thus, pathway-specific inhibition in FF and FB circuits (Shao and Burkhalter, 1996) may result from different subcellular distributions, convergences of inputs to individual PV neurons, or outputs from different size pools of PV neurons (Yamashita et al., 2003).

\section{PV, CR, and SOM neurons account for the majority of GABAergic targets in layer $2 / 3$}

Together, PV, CR, and SOM neurons account for $\sim 75 \%$ of all GABAergic neurons in layer 2/3 (Gonchar and Burkhalter, 1997) and represent $11.6-16.5 \%$ of synaptic targets of FF and FB axons. Although these are minimal estimates, because of incomplete staining of dendrites, they are similar to the total asymmetric input $(10-14 \%)$ to all GABAergic neurons identified by GABA immunogold labeling in cat and rat visual cortex (Lowenstein and Somogyi, 1991; Johnson and Burkhalter, 1996). This suggests that postembedding staining underestimates the number of GABAergic targets of FF and FB axons (Gonchar and Burkhalter, 1999a).

\section{Pathway-specific input to CR neurons in layer 1}

Layer 1 of rat cerebral cortex contains only GABAergic inhibitory neurons, of which $\sim 20 \%$ express CR (Gonchar and Burkhalter, 1997, 1999b; Hestrin and Armstrong, 1996; Chu et al., 2003). Inputs to layer 1 of rat cerebral cortex arise from thalamus, basal forebrain, brainstem, deep cortical layers, and higher cortical areas (Bear et al., 1985; Herkenham, 1986; Burkhalter, 1989; Coogan and Burkhalter, 1993; Mitchell and Cauller, 2001; Llinas et al., 2002). Of all these inputs, 5\% form synapses on CR neurons which is approximately four times less than FB inputs. Although these results suggest that CR neurons are selectively targeted by FB axons, it is surprising that stimulation of rat layer 1 rarely evokes inhibitory responses in layer $2-5$ pyramidal neurons (Cauller and Connors, 1994; Larkum and Zhu, 2002; Chu et al., 2003). When postsynaptic inhibition was observed, membrane hyperpolarization occurred close to the soma, suggesting that it was mediated by descending connections from layer 1 (Cauller and Connors, 1994). More frequently, however, inputs from layer 1 strongly depolarized apical dendrites of layer 2-5 pyramidal neurons (Cauller and Connors, 1994; Larkum and Zhu, 2002). These depolarizing responses had long onset latencies, slow rise times, and slow decays that differed from the fast sequence of excitation and inhibition elicited by stimulation of layer 6 (Cauller and Connors, 1994). This suggests that slow depolarizing responses in layer $2 / 3$ pyramidal neurons might result from disinhibition of distal dendrites by a synaptically interconnected network of inhibitory cells in layer 1 (Chu et al., 2003) that may involve CR neurons (this study). Similar to CR cells in layer 2/3 (Gonchar and Burkhalter, 199b), CR neurons in layer 1 may have a strong preference for innervating other layer $1 \mathrm{CR}$ neurons.

\section{Speculations on role of FB inputs to different layers}

Pyramidal neurons in layer $2 / 3$ of rat area 17 that project to area LM receive monosynaptic excitatory $\mathrm{FB}$ input to proximal dendrites in layer $2 / 3$ and to distal dendrites in layer 1 (Johnson and Burkhalter, 1997). Our results suggest that additional FB input to these neurons may derive from disynaptic connections via PV neurons in layer $2 / 3$ and CR neurons in layer 1 . In rodent visual cortex, FB synapses onto PV neurons are small and preferentially located on thin dendrites (Gonchar and Burkhalter, 1999a;
Yamashita et al., 2003). This organization of inputs may make inhibitory outputs of PV neurons ineffective in suppressing spike firing (Shao and Burkhalter, 1999) and conducive to backpropagation of action potentials (Larkum et al., 1999). Unlike inputs to layer 2/3, FB inputs to layer 1 are strongly linked to CR neurons, which may participate in an interconnected network of inhibitory neurons (Chu et al., 2003) whose disinhibitory output may depolarize distal pyramidal cell dendrites. Dendritic depolarization and coincident backpropagation of action potentials may increase the frequency of somatic firing (Larkum et al., 1999; Larkum and Zhu, 2002). This suggests the hypothesis that layerspecific connections with different types of GABAergic neurons may enable layer $2 / 3$ pyramidal neurons to associate $\mathrm{FB}$ inputs from higher areas with coincident responses to afferent visual input.

\section{References}

Anderson JC, Martin KAC (2002) Connection from cortical area V2 to MT in macaque monkey. J Comp Neurol 443:56-70.

Anderson JC, Binzegger T, Martin KAC, Rockland KS (1998) The connection from cortical area V1 to V5: a light and electron microscopic study. J Neurosci 18:10525-10540.

Bear MF, Carnes KM, Ebner FE (1985) An investigation of cholinergic circuitry in cat striate cortex using acetylcholinesterase histochemistry. J Comp Neurol 234:411-430.

Beaulieu C (1993) Numerical data on neocortical neurons in adult rat with special reference to the GABA-population. Brain Res 609:284-292.

Braitenberg V, Schüz A (1998) Cortex: statistics and geometry of neuronal connectivity, pp 99-101. Berlin: Springer.

Burkhalter A (1989) Intrinsic connections of rat primary visual cortex: laminar organization of axonal projections. J Comp Neurol 279:171-186.

Cauller LJ, Connors BW (1994) Synaptic physiology of horizontal afferents to layer 1 in slices of rat SI neocortex. J Neurosci 14:751-762.

Chu Z, Galarreta M, Hestrin S (2003) Synaptic interactions of late-spiking neocortical neurons n layers 1. J Neurosci 23:96-102.

Collins CE, Lyon JH, Kaas JH (2003) Responses of neurons in the middle temporal visual area after long-standing lesions of the primary visual cortex in adult New World monkeys. J Neurosci 23:2251-2264.

Coogan TA, Burkhalter A (1993) Hierarchical organization of areas in rat visual cortex. J Neurosci 13:3749-3772.

Crist RE, Li W, Gilbert CD (2001) Learning to see: experience an attention in primary visual cortex. Nat Neurosci 4:519-525.

Dantzker JL, Callaway EM (2000) Laminar sources of synaptic input to cortical inhibitory interneurons and pyramidal neurons. Nat Neurosci 3:701-707.

Das A, Gilbert CD (1999) Topography of contextual modulations mediated by short-range interactions in primary visual cortex. Nature 399:655-661.

Domenici L, Harding GW, Burkhalter A (1995) Patterns of synaptic activity in forward and feedback pathways within rat visual cortex. J Neurophysiol 74:2649-2664.

DeFelipe J (2001) Cortical interneurons: from Cajal to 2001. Prog Brain Res 136:215-238.

DeFelipe J, Gonzalez-Albo MC, DelRio MR, Elston GN (1999) Distribution and pattern of connectivity of interneurons containing calbindin, calretinin and parvalbumin in visual areas of the occipital and temporal lobes of the Macaque monkey. J Comp Neurol 412:515-526.

Feany MB, Lee S, Edwards RH, Buckley KM (1992) The synaptic vesicle protein SV2 is a novel type of transmembrane transporter. Cell 70:861-967.

Fisken RA, Garey LJ, Powell TPS (1975) The intrinsic, association and commissural connections of area 17 of the visual cortex. Philos Trans R Soc Lond B Biol Sci 272:487-536.

Girard P, Bullier J (1989) Visual activity in area V2 during reversible inactivation of area 17 in macaque monkey. J Neurophysiol 62:1287-1302.

Gonchar Y, Burkhalter A (1997) Three distinct families of GABAergic neurons in rat visual cortex. Cereb Cortex 7:347-358.

Gonchar Y, Burkhalter A (1999a) Differential subcellular localization of forward and feedback interareal inputs to parvalbumin expressing GABAergic neurons in rat visual cortex. J Comp Neurol 406:346-360. 
Gonchar Y, Burkhalter A (1999b) Connectivity of GABAergic calretininimmunoreactive neurons in rat primary visual cortex. Cereb Cortex 9:683-696.

Gulyás A, Megías M, Emri Z, Freund TF (1999) Total number and ratio of excitatory and inhibitory synapses converging onto single interneurons of different types in the CAl area of the rat hippocampus. J Neurosci 19:10082-10097.

Gupta A, Wang Y, Markram H (2000) Organizing principles for a diversity of GABAergic interneurons and synapses in the neocortex. Science 287:273-278.

Herkenham M (1986) New perspectives on the organization and evolution of nonspecific thalamocortical projections. In: Cerebral Cortex, Vol 5 (Jones EG, Peters A, eds), pp 403-445. New York: Plenum.

Hestrin S, Armstrong WE (1996) Morphology and physiology of cortical neurons in layer I. J Neurosci 16:5290-5300.

Hupé JM, James AC, Payne BR, Lomber SG, Girard P, Bullier J (1998) Cortical feedback improves discrimination between figure and background by V1, V2 and V3 neurons. Nature 394:784-787.

Hupé JM, James AC, Girard P Lomber SG, Payne BR, Bullier J (2001) Feedback connections act on the early part of the response in monkey visual cortex. J Neurophysiol 85:134-145.

Johnson RR, Burkhalter A (1994) Evidence for excitatory amino acid neurotransmitters in forward and feedback corticocortical pathways within rat visual cortex. Eur J Neurosci 6:272-286.

Johnson RR, Burkhalter A (1996) Microcircuitry of forward and feedback connections within rat visual cortex. J Comp Neurol 368:383-398.

Johnson RR, Burkhalter A (1997) A polysynaptic feedback circuit in rat visual cortex. J Neurosci 17:7129-7140.

Kawaguchi Y, Kubota Y (1997) GABAergic cell subtypes and their synaptic connections in rat frontal cortex. Cereb Cortex 7:476-486.

Larkum ME, Zhu JJ (2002) Signaling of layer 1 and whisker-evoked $\mathrm{Ca}^{2+}$ and $\mathrm{Na}^{+}$action potentials in distal and terminal dendrites of rat neocortical pyramidal neurons in vitro and in vivo. J Neurosci 22:6991-7005.

Larkum ME, Zhu JJ, Sakmann B (1999) A new cellular mechanism for coupling inputs arriving at different cortical layers. Nature 398:338-341.

Lee TS, Yang CFY, Romero RD, Mumford D (2002) Neural activity in early visual cortex reflects behavioral experience and higher-order perceptual saliency. Nat Neurosci 5:589-597.

Llinas RR, Leznik E, Urbano FJ (2002) Temporal binding via cortical coincidence detection of specific and nonspecific thalamocortical inputs: a voltage-dependent dye-imaging study in mouse brain slices. Proc Natl Acad Sci USA 99:449-454.

Lowenstein PR, Somogyi P (1991) Synaptic organization of cortico-cortical connections from primary visual cortex to the posteromedial lateral suprasylvian visual area in the cat. J Comp Neurol 310:253-266.
McDonald CT, Burkhalter A (1993) Organization of long-range inhibitory connections within rat visual cortex. J Neurosci 13:768-781.

Meskenaite V (1997) Calretinin-immunoreactive local circuit neurons in area 17 of the cynomolgus monkey, Macaca fascicularis. J Comp Neurol 379:113-132.

Mitchell BD, Cauller LJ (2001) Corticocortical and thalamocortical projections to layer I of the frontal neocortex in rats. Brain Res 921:68-77.

Peters A, Palay SL (1996) The morphology of synapses. J Neurocytol 25:687-700.

Peters A, Palay SL, Webster HF (1991) Fine structure of the nervous system. New York: Oxford UP.

Sandell JH, Schiller PH (1982) Effects of cooling area 18 on striate cortex in the squirrel monkey. J Neurophysiol 48:38-48.

Schiller PH, Malpeli JG (1977) The effect of striate cortex cooling on area 18 cells in monkey. Brain Res 126:366-369.

Shao Z, Burkhalter A (1996) Different balance of excitation and inhibition in forward and feedback circuits of rat visual cortex. J Neurosci 16:7353-7365.

Shao Z, Burkhalter A (1999) Role of $\mathrm{GABA}_{\mathrm{B}}$ receptor-mediated inhibition in reciprocal interareal pathways of rat visual cortex. J Neurophysiol 81:1014-1024.

Staiger J, Zilles K, Freund TF (1996) Distributions of elements postsynaptic to ventroposteromedial thalamic projections in layer IV of rat barrel cortex. Eur J Neurosci 8:2273-2285

Thomson AM, West DC, Wang Y, Bannister AP (2002) Synaptic connections and small circuits involving excitatory and inhibitory neurons in layers 2-5 of adult rat and cat neocortex: triple intracellular recordings and biocytin labeling in vitro. Cereb Cortex 12:936-953.

Traczy-Hornoch C, Martin KA, Stratford KJ, Jack JJB (1999) Intracortical excitation of spiny neurons in layer 4 of cat striate cortex. Cereb Cortex 9:833-843.

Wang Y, Gupta A, Toledo-Rodriguez M, Wu CZ, Markram H (2002) Anatomical, physiological, molecular and circuit properties of nest basket cells in the developing somatosensory cortex. Cereb Cortex 12:395-410.

Weinberg RJ, Van Eyck SJ (1991) A tetramethyl benzidine/tungstate reaction for horseradish peroxidase histochemistry. J Histochem Cytochem 39:1143-1148.

West MJ, Slomianka L, Gundersen HJ (1991) Unbiased stereological estimation of the total number of neurons in the subdivisions of the rat hippocampus using the optical fractionator. Anat Rec 231:482-497.

Yamashita A, Valkova K, Gonchar Y, Burkhalter A (2003) Rearrangement of synaptic connections with inhibitory neurons in developing mouse visual cortex. J Comp Neurol 464:426-437. 